\title{
NEGLIGENCIA AL CUIDADO DENTAL. REVISIÓN DE LA LITERATURA.
}

\section{DENTAL NEGLECTE.LITERATURE REVIEW.}

\author{
Matute-Riofrío Caridad ${ }^{1}$, Barzallo-Pérez Juan ${ }^{2}$,Villavicencio-Caparó Ebingen ${ }^{3}$ \\ ${ }^{1}$ Odontóloga General.Consulta Privada.Ecuador. \\ 2 Odontólogo Rural del Mnisterio de Salud Pública del Ecuador. \\ ${ }^{3}$ Coordinador de Investigación de la Carrera de Odontología de la Universidad Católica de Cuenca.Ecuador. \\ * carimatute@hotmail.com
}

\begin{abstract}
Resumen
Se realizó una revisión bibliográfica con el fin de recopilar información científica acerca de lo descrito por los autores en cuanto a la negligencia al cuidado dental con el propósito de obtener datos estadísticos que comprueben la presencia de negligencia al tratamiento odontológico para el mantenimiento de una adecuada salud de la cavidad bucal; se analizó este fenómeno que afecta a la población en general, y se obtuvieron datos sobresalientes sobre los factores de riesgo más comunes, con una escala de valoración (OR y p), dándonos como resultado el principal causante el lugar de residencia; se da a conocer información sobre la escala de valoración utilizada escala dental de negligencia(DNS) la cual determina si existe o no negligencia en el individuo. Conclusión la negligencia al cuidado dental es un fenómeno que ha permanecido a lo largo del tiempo por la falta de estudios certeros que con lleven a un tratamiento adecuado por parte de los profesionales, que provocan una deficiente salud bucal y empeora la calidad de vida de los pacientes.
\end{abstract}

Palabras clave: Negligencia dental , escala.

\begin{abstract}
A bibliographic review was carried out in order to collect scientific information about what the authors described regarding neglect of dental care in order to obtain statistical data that prove the presence of neglect of dental treatment for the maintenance of an adequate oral cavity health; we analyzed this phenomenon that affects the population in general, and we obtained outstanding data about the most common risk factors, with an assessment scale (OR and p), resulting in the main cause of the place of residence; information is provided on the scale of assessment used for neglect (DNS) dental scale which determines whether or not there is negligence in the individual. Conclusion neglect of dental care is a phenomenon that has remained over time due to the lack of accurate studies that lead to an adequate treatment by professionals, which cause poor oral health and worsens the quality of life of the patients. patients.
\end{abstract}

Key words: Dental Negligence, scale.

\section{INTRODUCCIÓN}

Negligencia al cuidado dental hace referencia al comportamiento y las actitudes con probables consecuencias perjudiciales para la salud bucal del individuo. ${ }^{1}$ Es el incumplimiento de la debida atención odontológica para el mantenimiento de la cavidad bucal. ${ }^{2}$ Thomson WM, Spencer AJ y cols. Definen la negligencia al cuidado dental como falta de tomar precauciones para mantener la salud bucal, incapacidad para obtener la atención dental necesaria y la negligencia física de una cavidad dental. ${ }^{3,4}$ Según Sarri G. el término negligencia al cuidado dental es la ausencia de prevención para la enfermedad bucal que resulta en malas condiciones dentales.

La negligencia al cuidado dental se clasifica en:

- Negligencia activa: Incumplimiento intencional de los padres o tutores para cumplir con sus responsabilidades de cuidado.

- Negligencia pasiva: Incumplimiento involuntario de los padres o tutores para cumplir con su responsabilidad, conocimiento, enfermedad, debilidad, finanzas o la falta de conocimiento del apoyo comunitario disponible.

- Negligencia al autocuidado dental: Discapacidad física, mental o de desarrollo que impide la satisfacción propia de las necesidades dentales. ${ }^{5}$ 


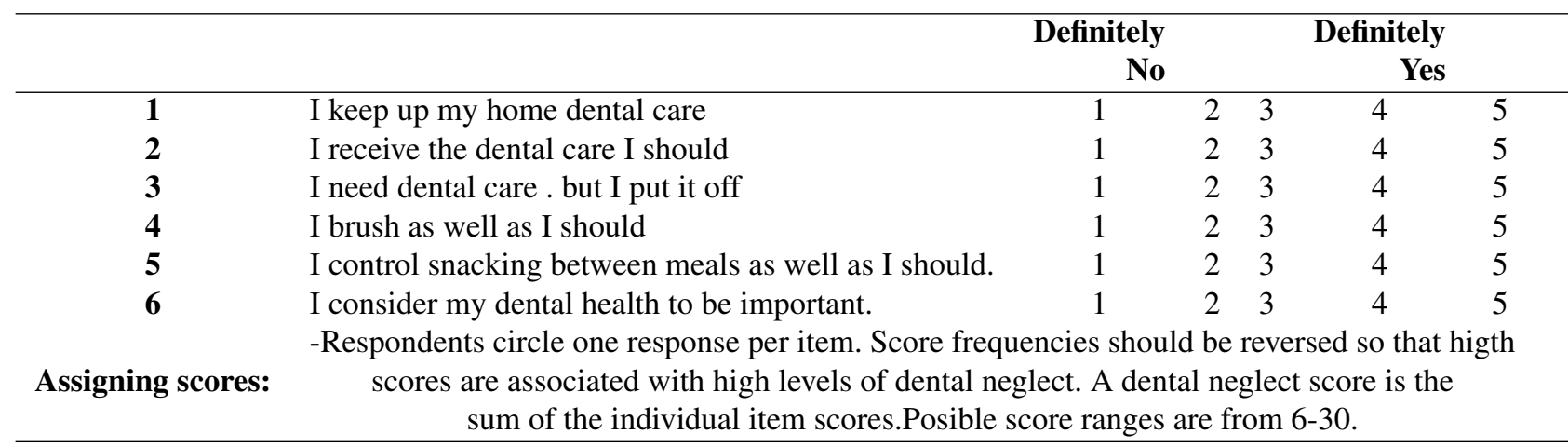

Tabla.1: Escala de Negligencia al cuidado Dental.

La negligencia dental tiene un origen étnico, cultural, religioso y educativo ${ }^{6}$ y se vincula con el analfabetismo entre la clase socio-económica baja. ${ }^{2}$ Se relacionada con una mala salud bucal, la falta de exámenes de rutina y periodos de tiempo prolongado desde la última cita con el odontólogo ${ }^{7}$ Está asociada con una serie de resultados negativos en la salud bucal y el funcionamiento general del sistema estomatognático. ${ }^{2}$

La prevención de la enfermedad bucal es sin duda muy eficaz, eficiente, adecuada y aceptable, es un hábito que conduce a una mejor salud bucal ${ }^{2}$ La caries dental, enfermedad periodontal y otras condiciones orales, si no se tratan a tiempo pueden provocar dolor, infección y pérdida de funciones, afectando negativamente el aprendizaje, la comunicación, la nutrición y otras actividades necesarias para el crecimiento normal y el desarrollo del individuo. La falta de buscar u obtener atención dental adecuada conlleva factores como el aislamiento familiar, la falta de financiamiento, la ignorancia de los padres, o la falta de valor percibido hacia salud bucal. ${ }^{3}$

El cuidado dental debe centrarse en el alivio del dolor y otros síntomas, seguidos por la restauración apropiada de la función y el aspecto. Se han recomendado los siguientes principios para la respuesta del equipo dental preventivo: plantear inquietudes a los padres y cuidadores, explicar los cambios que se necesitan, ofrecer apoyo, llevar registros, mantener contacto permanente con los padres o cuidadores y revisar el progreso. ${ }^{5}$

La educación sobre la salud bucodental debe incorporarse desde el principio al currículo educativo, para que se pueda enseñar a los jóvenes las enfermedades dentales que puedan padecer si no toman precauciones adecuadas desde la primera infancia.La higiene bucal está totalmente relacionada con el aspecto conductual de la persona afectada. Con el fin de cuidar de sus dientes una persona necesita tener una actitud positiva hacia la salud bucodental. ${ }^{2}$

\section{ESTADO DEL ARTE.}

\subsection{Escalas para medir la negligencia al cuidado dental}

Para medir la negligencia al cuidado dental se realizan encuestas como la Escala Dental de Negligencia (DNS), que evalúa hasta qué punto un individuo se preocupa por sus dientes, recibe atención dental profesional y cree que la salud oral es importante.

Se realiza una encuesta DNS, la cual contiene un cuestionario con las siguientes declaraciones:

1) ¿Mantengo mi cuidado dental en el hogar?

2) ¿Recibo el cuidado dental que debo?

3) ¿Necesito el cuidado dental, pero lo pospongo?

4) ¿Me cepillo los dientes tan bien como debería?

5) ¿Me controlo de comer entre comidas?

6) ¿Considero mi salud dental importante?

Escala mediante la cual se evaluó la negligencia dental capturando las respuestas personales, y utilizando una escala de Likert que va desde uno ("definitivamente no") a cinco ("definitivamente sí). ${ }^{4}$ Tabla 1.

\subsubsection{Validez de la escala.}

La sensibilidad y especificidad de un instrumento debe ser medida para verificar la validez del mismo, se usa la prueba Alpha de Cronbach para medirlo según Thomson en el año 2000 las respuestas de la encuesta se sumaron para crear una escala de negligencia la cual obtuvo un Alpha de Cronbach de 0,71.

Otra evaluación del instrumento fue realizada por E. Skaret logrando un Alpha de Cronbach de 0,57 en una muestra poblacional de 1309 personas.

Trilbi Coolidge en el año 2009, por medio de Alpha de Cronbach determinó la confiabilidad de la escala DNS en 117 participantes con un resultado de 0,60. Tabla 3.

\subsection{Factores de Riesgo de Negligencia al cuidado dental.}

Existen diferentes factores de riesgo que pueden desencadenar negligencia al cuidado dental, siendo algunos más 


\begin{tabular}{|c|c|c|c|c|c|}
\hline \multicolumn{6}{|c|}{ Tabla 2.- Factores de riesgo } \\
\hline Referencia & & $\mathbf{O R}$ & $\mathbf{p}$ & $\%$ & Significancia \\
\hline \multirow[t]{5}{*}{ Sarri G 2012} & Género : Masculino & 1 & & & FR:No Significativo \\
\hline & Femenino & 1.16 & $0.28 *$ & & \\
\hline & Lugar de Residencia & 1.12 & & & FR: Significativo \\
\hline & Última visita al odontólogo: Hace un año o menos & 1 & & & FR: No Significativo \\
\hline & Más de un año & 4.20 & $<0.001 *$ & & FR:Significativo \\
\hline \multirow[t]{8}{*}{ McGrath 2007} & Edad & & $<0.001 * * *$ & & FR: Significativo \\
\hline & Género & & $>0.05 * *$ & & FR:No Significativo \\
\hline & Educación & & $<0.01 * * *$ & & FR: Significativo \\
\hline & Ingresos & & $<0.01 * * *$ & & FR: Significativo \\
\hline & Lugar de residencia & & $>0.05 * *$ & & FR:No Significativo \\
\hline & Auto reporte de salud bucal & & $<0.001 * * *$ & & FR: Significativo \\
\hline & Uso de prótesis & & $<0.001 * *$ & & FR: Significativo \\
\hline & Número de dientes presentes & & $<0.01 *$ & & FR:Significativo \\
\hline \multirow[t]{7}{*}{ Stiefel D.1990 } & Género & & $0.69 *$ & & FR:No Significativo \\
\hline & Edad & & $0.55 *$ & & FR:No Significativo \\
\hline & Grupo Familiar & & $0.94 *$ & & FR:No Significativo \\
\hline & Ingresos & & $0.87 *$ & & FR:No Significativo \\
\hline & Fuente de ingresos & & $0.67 *$ & & FR:No Significativo \\
\hline & Nivel de educación materno & & $<0.05^{*}$ & & FR: Significativo \\
\hline & Visita al odontólogo en los últimos 2 años. & & $<0.05^{*}$ & & FR: Significativo \\
\hline \multirow[t]{6}{*}{ Skaret E.2007 } & Auto reporte de salud bucal & 3,25 & & & FR: Significativo \\
\hline & Razon principal de la ultima consulta & 2,26 & & & FR: Significativo \\
\hline & Numero de dientes sanos & 2,24 & & & FR: Significativo \\
\hline & Frecuencia de ingesta de bebidas azucaradas & 2.06 & & & FR: Significativo \\
\hline & Servicio dental usado & 2,17 & & & FR: Significativo \\
\hline & Uso de hilo dental o palillo dental & 1,56 & & & FR: Significativo \\
\hline
\end{tabular}

FR: Factor de Riesgo *OR **T de Student *** ANOVA.

\begin{tabular}{llcc}
\hline \multicolumn{1}{c}{ Referencia } & \multicolumn{1}{c}{ Tabla 3.-Estudios de Confiablidad } \\
Pais & p & n \\
\hline E. Skaret 2007 & Noruega & 0.57 & 1309 \\
T.Coolidge 2009 & Estados Unidos & 0.60 & 117 \\
Thomson 2000 & Nueva Zelanda & 0.71 & 973 \\
\hline
\end{tabular}

$\mathrm{p}=$ Cronbach's Alpha

relevantes que otros. (Tabla2) McGrath C. expone la respuesta de los individuos, relacionando la calidad de vida con la salud oral (OHQOL) Tabla 2. ${ }^{8}$ Los estudios realizados en diversas poblaciones exponen los siguientes factores de riesgo como los más comunes. En la Tabla 3 podemos observar los factores de riesgo que causan negligencia al cuidado dental, los más frecuentes según el estudio de Sarri G en el 2012; los factores de riesgo significativos son: lugar de residencia, y última visita al odontólogo. McGrath C. en el año 2007 menciona edad, educación, ingresos, autoreporte de salud bucal, uso de prótesis. ${ }^{8,9}$

Stiefel D. en 1990 señala que el nivel de educación de la madre, y la visita al odontólogo en los últimos dos años son valores significativos con un $\mathrm{p}<0,05{ }^{10}$ Skaret E. en al año 2007, expresa que el auto reporte de salud bucal OR 3,25 ; la razón principal de la última consulta OR 2,26; el número de dientes sanos OR 2,24, la frecuencia de ingesta de bebidas azucaras OR 2,06; servicio dental usado OR 2,17; y uso de hilo dental o palillo de dientes OR 2,56; representan un Factor significativo de Riesgo. ${ }^{11}$

\subsection{Manejo de la Negligencia al Cuidado Dental.}

En la búsqueda de información no se encontraron ensayos clínicos que respalden intervenciones para el manejo de este tipo de pacientes adultos, sin embargo, si se encontró artículos de opinión y de posición respecto a cómo debiera ser el manejo de la negligencia, como por ejemplo en el artículo del Dr. Kumar, que recomienda tre etapas de intervención cuando se detecta Negligencia al cuidado dental en niños: Primero el trabajo en equipo para la prevención de enfermedades bucales, segundo la gestión preventiva multidisciplina- 
ria -dado que la negligencial cuidado dental siempre se ve acompañada de negligencia al cuidado en general- y tercero la referencia del niño a las agencias de protección infantil. ${ }^{5}$ Por otro lado la sociedad de Odontopediatría de California sugiere que los riesgos y beneficios del tratamiento dental deben ser explicados a los padres y estos deben saber que los analgésicos apropiados y procedimientos anestésicos se utilizarán para garantizar la la comodidad del niño durante los procedimientos dentales. $\mathrm{Si}$, a pesar de estos esfuerzos, el padre no puede obtener la terapia, el caso debe ser reportado a la agencia apropiada de servicios de protección infantil. ${ }^{12}$

\section{DISCUSIÓN}

La negligencia al cuidado dental es una condición, que cual afecta al adecuado auto cuidado de la salud bucal incumpliendo el mantenimiento de esta. Según Sarri G. el lugar de residencia es un factor de riesgo mientras que McGrath C afirmó que no lo es. ${ }^{8,9}$ Sarri G, y Stiefel D coincidieron que la última visita al odontólogo es un factor de riesgo de importancia. ${ }^{8,10}$ McGrath C manifestó que la edad es un factor de riesgo significativo, y por lo contrario Stiefel D sostuvo que no. ${ }^{9,10}$ Según McGrath C y Stiefel D la educación fue un factor de riesgo. ${ }^{9}$ Para McGrath $\mathrm{C}$ el ingreso salarial fue un factor de riesgo significativo mientras que Stiefel D afirmó lo contrario. ${ }^{9,10}$ McGrath C y E Skaret, confirmaron que el autoreporte de salud bucal es un factor de riesgo significativo. ${ }^{9,11}$ Mc Garth C. y Skaret E. afirman que los dientes presentes son un factor de riesgo. ${ }^{9,11}$ Según Mc Grath C el uso de prótesis es un factor de riesgo. ${ }^{9}$

E. Skaret afirmó que el servicio dental usado, el uso de hilo dental o palillo de dientes y la razón principal de la última consulta son factores de riesgo. ${ }^{11}$ E. Skaret, T Coolidge y Thomson WM, presentaron valores similares en cuanto a la confiabilidad de la escala DNS, pero se debe regir principalmente al valor descrito por E. Skaret ya que su valor muestral fue de 1309 personas, lo que nos indicó un mayor intervalo de confianza con un resultado de Alpha de Cronbach de 0,57. $1,7,11$

\section{CONCLUSIÓN}

La negligencia al cuidado dental, es una condición que puede afectar ala salud bucal de manera crítica. Existe una escala suficientemente validada para poder medirla, se conocen algunos de los factores de riesgo para esta y no hay en la literatura revisada intervenciones que puedan disminuirla.

Conflicto de intereses y financiamiento Los autores declaran no tener conflicto de intereses, haber cumplido con los requisitos de autoría y haber autofinanciado este artículo.

Agradecimiento al Dr.Thomson WM. por su comunicación vía e-mail para despejar dudas.

\section{Referencias}

1 Thomson WM, Spencer AJ, Gaughwin A. Testing a child dental neglect scale in South Australia. Community Dent Oral Epidemiol. 1996 Oct;24(5):351-6. PubMed PMID: 8954223.

2 Sarkar P, Dasar P, Mishra P. Impact of Dental Neglect Scale on Oral Health Status Among Different Professionals in Indore City-A Cross- Sectional Study.. [Online].; 2015 Oct; 9.

3 Mathur A, Aggarwal V. Dental Neglect affecting oral health status in India. International Journal of Pediatric Research. 2016 Enero; 2(1)

4 Jamieson L TM. Dental health, dental neglect and use of services in an adult Dunedin population sample. New Zeland dental journal. [Online].; 2002.

5 Kumar A, Manjunath P, Sowmia KR. Dental neglect a review, Journal of dental Sciences. 2015 Mayo; 3(1):19 [Online].; 2015

6 Ramazani N. Child Dental Neglect: A Short Review. International Journal of High Risk Behaviors \& Addiction. 2014;3(4):e21861. doi:10.5812/ijhrba.21861.

7 Coolidge T, Heima M, Johnson EK, Weinstein P. The Dental Neglect Scale in adolescents. BMC Oral Health. 2009 Jan 5;9:2. doi: 10.1186/1472-6831-9-2. PubMed PMID: 19123953; PubMed Central PMCID: PMC2627830.

8 McGrath C, Sham AS, Ho DK, Wong JH. The impact of dental neglect on oral health: a population based study in Hong Kong. Int Dent J. 2007 Feb;57(1):3-8. PubMed PMID: 17378343.

9 Sarri G, Evans P, Stansfeld S, Marcenes W. A schoolbased epidemiological study of dental neglect among adolescents in a deprived area of the UK. Br Dent J. 2012 Nov;213(10):E17. doi: 10.1038/sj.bdj.2012.1042. Erratum in: Br Dent J.2012 Dec;213(12):615. PubMed PMID: 23175099.

10 Stiefel D. A comparision of the oral health of person with and without chronic mental illnes in comunity setting. [Online].; 1990.

11 Skaret E, Astrøm AN, Haugejorden O, Klock KS, Trovik TA. Assessment of the reliability and validity of the Dental Neglect Scale in Norwegian adults.Community Dent Health. 2007 Dec;24(4):247-52. PubMed PMID: 18246843.

12 California Society of Pediatric Dentists. Dental neglect:when to report. California Pediatrician 1989;Fall:31-2

Recibido: 16 de Marzo de 2018

Aceptado: 9 de Julio de 2018 